\title{
Targeted Therapies Overcoming Endocrine Resistance in Hormone Receptor-Positive Breast Cancer
}

\author{
Katrin Almstedt Marcus Schmidt \\ Department of Obstetrics and Gynaecology, Johannes Gutenberg University, Mainz, Germany
}

\author{
Keywords \\ Breast cancer · Metastasized · Endocrine therapy · p16 . \\ Everolimus · Palbociclib
}

\section{Summary}

Breast cancer is a heterogeneous disease with different molecular subtypes. Most tumours are hormone receptor positive (luminal subtype) with potential endocrine responsiveness. Endocrine therapy is commonly used in these patients. Disease progression caused by endocrine resistance represents a significant challenge in the treatment of breast cancer. To understand the mechanisms of resistance of long-term oestrogen-deprived breast cancer cells, it is important to focus on cross-talk between steroid receptor signalling and other growth factor receptors and intracellular pathways. (Pre-)clinical trials showed that co-targeting these pathways can restore endocrine sensitivity. The focus of the current review is on the intracellular $\mathrm{PI} / \mathrm{K} / \mathrm{AKT} / \mathrm{mTOR}$ signalling pathway and cyclin-dependant kinases (CDKs) in oestrogen receptor (ER)-positive breast cancer. Study results clearly show that both inhibition of the PI3K/AKT/mTOR pathway and CDK4/6 are promising ways to improve the efficacy of endocrine treatment in ER-positive breast cancer patients with comparably few side effects. Further clinical trials are needed to identify the patient population who would benefit most from a dual inhibition.

\section{Introduction}

Breast cancer is a heterogeneous disease and the different treatment strategies depend primarily on the molecular subtype [1]. Approximately $70 \%$ of breast cancers express oestrogen receptor (ER) $\alpha$ and are endocrine responsive. However, the possibility of altered tumour characteristics with a switch of hormone receptor (HR) expression has to be considered [2]. Due to its high efficacy and favourable tolerability, endocrine therapy is the treatment of choice for patients with an advanced hormone receptor-positive tumour with the only exception of acute life threatening disease [3-5]. Over time, a large proportion of patients develop a primary/ de novo resistance or secondary/acquired resistance. It is, therefore, important to understand the pathways and cross-talk involved to determine a way to overcome the resistance mechanisms. A large number of publications deal with the cross-talk between steroid receptors (ER and progesterone receptor (PR)) and growth factor receptors (e.g. epidermal growth factor receptor (EGFR)/ human epidermal growth factor receptor (HER) [6], insulin-like growth factor receptor (IGFR) [7], and fibroblast growth factor receptor (FGFR) [8]) as well as the intracellular pathways (e.g. phosphatidylinositol 4,5-bisphosphate-3-kinase (PI3K)/protein kinase $\mathrm{B}(\mathrm{AKT}) /$ mammalian target of rapamycin (mTOR) signalling). This review focuses on the intracellular PI3K/AKT/mTOR signalling pathway and cyclin-dependant kinases (CDKs) as (indirect) activators of ligand-independent ER activation. Furthermore, the cross-talk between ER and HER2 as well as the therapeutic implications of this dual blockade are briefly outlined.

\section{HER2/EGFR}

Patients with HER2-overexpressing breast cancer are less responsive to endocrine therapy. It was hypothesized that endocrine resistance is due to a cross-talk between HER and HR pathways. Therefore, HER signalling was one of the first pathways analysed, also in

\section{KARGER \\ Fax +497614520714

Prof. Dr. med. Marcus Schmidt 
Fig. 1. Intracellular pathways involved in endocrine resistance. $\mathrm{CDK}=$ cyclin-dependent kinase, Deptor $=$ DEP domain-containing mTOR-interacting protein, EGFR = epidermal growth factor receptor, ER = oestrogen receptor, FGFR = fibroblast growth factor receptor, HER = human epidermal growth factor receptor, IGFR = insulin-like growth factor receptor, $\mathrm{mLST} 8=$ mammalian lethal with SEC13 protein $8, \mathrm{mTOR}=$ mammalian target of rapamycin/mechanistic target of rapamycin, mTORC1 $=$ mammalian target of rapamycin complex $1 /$ mechanistic target of rapamycin complex 1 , PI3K = phosphatidylinositol 4,5-bisphosphate3-kinase, PIP2 = phosphatidylinositol 4,5-bisphosphate, PIP3 = phosphatidylinositol 3,4,5-triphosphate, PRAS = proline-rich AKT1 substrate 1, Raptor $=$ regulatory-associated protein of $\mathrm{mTOR}, \mathrm{S} 6 \mathrm{~K} 1$ $=$ ribosomal protein $\mathrm{S} 6$ kinase beta-1, $4 \mathrm{EBP} 1=4 \mathrm{E}-$ binding protein 1 .

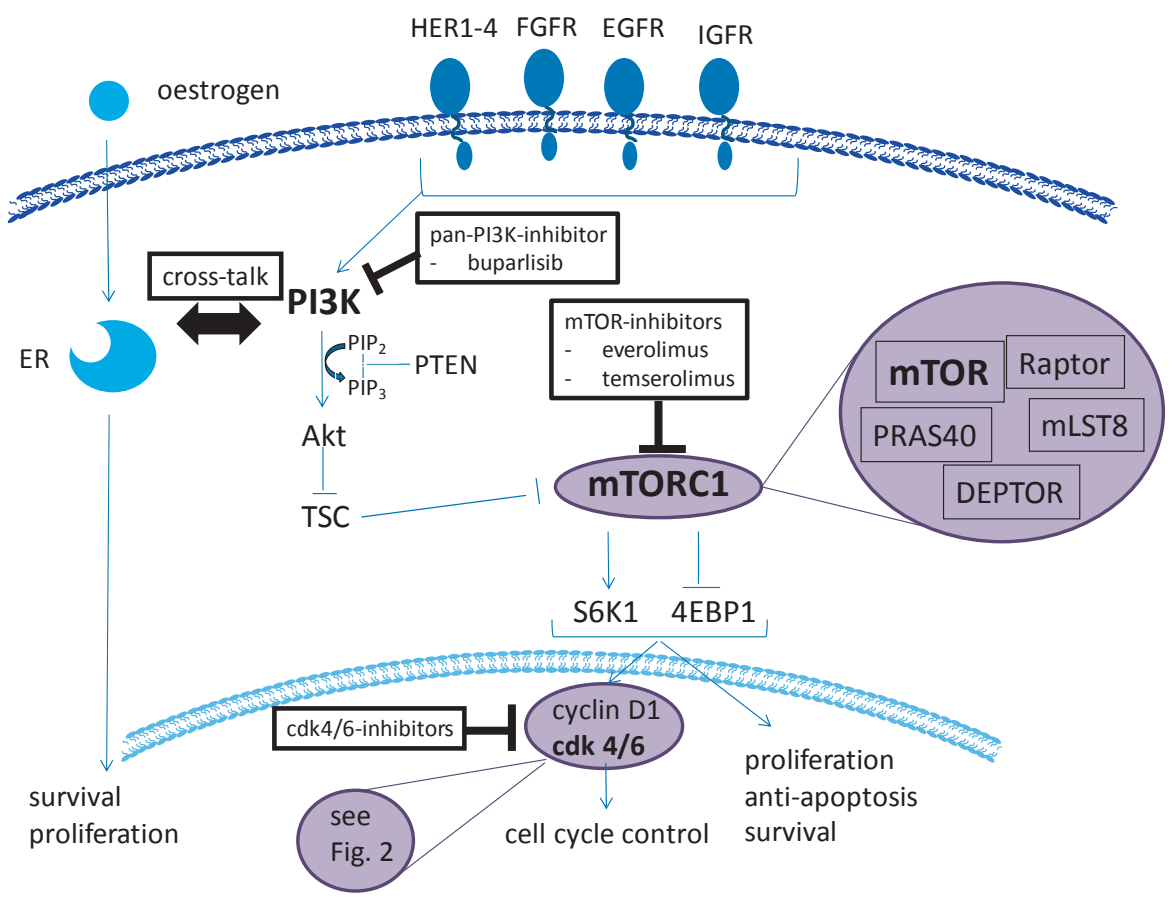

mTOR

HER-negative breast cancer. The HER family includes four tyrosine kinase receptors, HER1 (EGFR, ErbB1), HER2 (ErbB2), HER3 (ErbB3) and HER4 (ErbB4). The formation of homo- and heterodimers results in an activation of several downstream pathways such as the PI3K- or the mitogen-activated protein kinase (MAPK) pathway [9]. This dual blockade was examined in several clinical trials. The largest trial until now, a phase III study with 1,286 patients, compared daily letrozole ( $2.5 \mathrm{mg}$ orally) plus lapatinib (1,500 mg orally) versus letrozole alone in patients with ER-positive metastatic breast cancer [10]. This trial showed a significantly enhanced progression-free survival (PFS; primary endpoint, 8.2 vs. 3.0 months, hazard ratio (HR) $0.71,95 \%$ confidence interval (CI) $0.53-0.96$; $\mathrm{p}=0.019$ ) and response rate for patients with HER2-positive breast cancer. There was no benefit in PFS for HER2-negative tumours. The results of the TAnDEM-trail also demonstrated an advantage for co-targeting HER2 in endocrine-resistant breast cancer (median PFS 4.8 vs. 2.4 months, HR 0.63, 95\% CI 0.47-0.84, $\mathrm{p}=0.0016$; centrally confirmed HR-positive tumours 5.6 vs. 3.8 months, $\mathrm{p}=0.006$ ) [11]. In this phase III study, 207 patients with HER2- and HR-positive metastatic breast cancer were randomised to anastrozole $(1 \mathrm{mg} /$ day orally) with or without trastuzumab (4 mg/kg intravenous infusion on day 1 , then $2 \mathrm{mg} / \mathrm{kg}$ every week). However, in the CALGB 40302-study, no significant improvement for a dual inhibition was seen [12]. In this phase III study 295 patients with HR-positive advanced breast cancer, regardless of HER2 status, were randomised to fulvestrant (500 mg on day 1 followed by $250 \mathrm{mg}$ on days 15 and 28 and then every 4 weeks) and either daily lapatinib $(1,500 \mathrm{mg})$ or placebo (median PFS 4.7 vs. 3.8 months, HR 1.04, 95\% CI 0.82-1.33, $\mathrm{p}=0.37$. Taken together, these results indicate that dual blockade of ER and HER2 has the potential to improve outcome in patients with advanced ER- and HER2-positive but not HER2-negative breast cancer patients.
PI3K mutations are the most common alterations in ER-positive breast cancer cells [13] and an up-regulation of this pathway is frequently seen in long-term oestrogen-deprived breast cancer cells [14]. The PI3K/AKT/mTOR pathway has been well analysed in several cellular processes, including proliferation, apoptosis, angiogenesis and survival. The PI3K heterodimer, comprising a regulatory (p85) and a catalytic (p110) subunit, is regulated by different growth factor receptors, e.g. EGFR/ HER, IGFR, FGFR (fig. 1). $\mathrm{PIP}_{3}$ (phosphatidylinositol 3,4,5-triphosphate) is the catalytic product of the p110, which leads to a stimulation of the mTOR via phosphorylation/activation of protein kinase B/AKT (a serine/threonine kinase). mTOR is always present as a protein complex [15]. The mTORC 1 complex (fig. 1) is the target of rapamycin and rapamycin analogues, such as everolimus (inhibitor of the mTOR) or temsirolimus. In July 2012, the US Food and Drug Administration (FDA) [16] and the European Commission (EMA) approved everolimus for treating postmenopausal women with hormone receptor-positive/HER2-negative advanced breast cancer. The study that led to the approval was the BOLERO2-trial, a phase III study that included 724 patients and compared everolimus ( $10 \mathrm{mg} /$ day) plus exemestane $(25 \mathrm{mg} /$ day) to exemestane alone in postmenopausal women with advanced hormone receptor-positive breast cancer, whose cancer had either failed treatment with non-steroidal aromatase inhibitors in the metastatic disease or recurred in the adjuvant setting [17]. The results for the primary endpoint after a median follow-up of 18 months indicated that a co-inhibition of the compensatory pathway via everolimus led to a doubling of PFS (local assessment: 7.8 vs. 3.2 months, HR $0.45,95 \%$ CI $0.38-0.54, \mathrm{p}<0.0001$; central assessment: 11.0 vs. 4.1 months, HR $0.38,95 \%$ CI 0.31 to $0.48, \mathrm{p}<$ 
0.0001) [18]. The greatest effect was observed for the subgroup of first-line therapy for advanced breast cancer (local assessment: 11.5 vs. 4.1 months, HR $0.39,95 \%$ CI 0.25 to 0.62 ; central assessment: 15.2 vs. 4.2 months, HR 0.32, 95\% CI 0.18-0.57) [19]. Further evaluations with respect to the secondary endpoint demonstrated a numerically increased, but not significantly improved, overall survival (31.0 vs. 26.6 months, HR 0.89 , 95\% CI 0.73-1.10, $\mathrm{p}=0.14$ ) [20]. It was also recently shown for tamoxifen that cotargeting with everolimus led to restored sensitivity in endocrineresistant breast cancer. Therefore, the TAMRAD-trial, a phase IIstudy ( $\mathrm{n}=111$ ), analysed the addition of everolimus to tamoxifen (tamoxifen $20 \mathrm{mg} /$ day plus everolimus $10 \mathrm{mg} /$ day or tamoxifen alone $20 \mathrm{mg}$ /day) in postmenopausal women with advanced hormone receptor-positive breast cancer showing primary or secondary resistance to aromatase inhibitors [21]. The study results suggested an increased clinical benefit (primary endpoint, clinical benefit rate (CBR) $61 \%$ vs. $42 \%$; $95 \%$ CI, $47-74$ vs. $29-56$, $\mathrm{p}=0.045$ ), longer time to progression (8.6 vs. 4.5 months, $95 \%$ CI, $5.9-13.9$ vs. $3.6-8.7 ; \mathrm{p}=0.002)$ and reduced risk of death (55\%, HR 0.45; 95\% CI, 0.24-0.81; $\mathrm{p}=0.007)$. In particular, patients with a secondary endocrine resistance, defined as 6 months after stopping endocrine therapy in the adjuvant setting or at least 6 months after initiating endocrine treatment for metastatic breast cancer, achieved benefit from an additional inhibition with everolimus. A different approach was assessed in the Horizon study in which a mTOR inhibitor was used in the first-line setting to try to avoid endocrine resistance. This phase III study $(\mathrm{n}=1,112)$ evaluated the addition of temsirolimus $(30 \mathrm{mg} /$ day for 5 days every 2 weeks) to letrozole in the first-line setting in patients with hormone receptor-positive advanced breast cancer [22]. Most of the patients were endocrine therapy naive. However, the study was terminated at an early point by the data-monitoring committee because of a lack of efficacy in the interim analysis (PFS 9 vs. 8.9 months, HR 0.9, $95 \%$ CI 0.76-1.07, p = 0.25; overall response rate in both arms $27 \%$ ).

\section{PI3K/AKT}

In contrast to $\mathrm{mTOR}$ inhibitors, therapies against targets upstream in the PI3K/AKT/mTOR pathway have not yet been approved in breast cancer, with numerous questions still open. In this context, buparlisib (BKM120), a pan-class-PI3K-inhibitor, is currently being analysed in the study designs of the BELLE (Buparlisib Breast Cancer Clinical Evaluation) trials. The BELLE-2-trial, a phase III study, examined the efficacy of fulvestrant plus buparlisib in women with hormone receptor-positive, HER2-negative advanced breast cancer that was refractory to the treatment with aromatase inhibitors [23] and the phase III BELLE-3-study investigated whether fulvestrant plus buparlisib was able to restore the endocrine sensitivity after the treatment with an mTOR inhibitor [24]. Final study results are still pending.

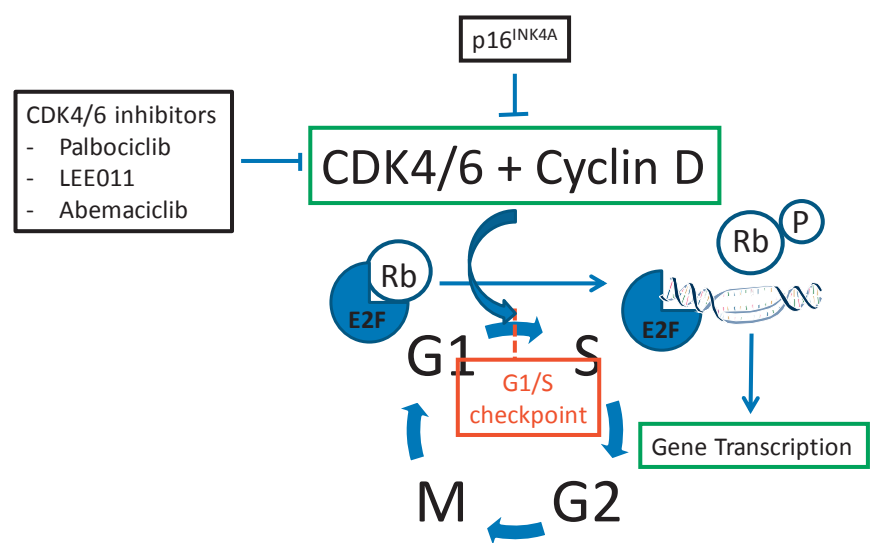

Fig. 2. Role of CDK4/6 in cell cycle regulation. $\mathrm{G} 1=$ gap1 phase, $\mathrm{G} 2$ = gap2 phase, E2F = E2 promoter binding factor, $\mathrm{M}=$ mitotic phase, $\mathrm{P}=$ phosphorylation, $\mathrm{p} 16=$ protein $16 / \mathrm{CDK}$ inhibitor $2 \mathrm{~A} /$ multiple tumour suppressor $1, \mathrm{Rb}=$ retinoblastoma protein, $\mathrm{S}=$ synthetic phase.

\section{CDK4/6}

Limitless replicative potential with disrupted cell-cycle regulation is one of the hallmarks of cancer [25]. Cells have to progress through the four phases of the cell cycle to divide and replicate: G1, $S$ phase (DNA synthesis), G2, and M phase (mitosis). The central players for regulation of the cell cycle are CDKs, a group of serine/ threonine kinases that form active heterodimeric complexes following binding to cyclins, thereby facilitating the transition of the cell cycle [26]. CDK4/6 is the key regulator of the G1-S transition. In complex with cyclin $\mathrm{D}, \mathrm{CDK} 4 / 6$ phosphorylates retinoblastoma protein $(\mathrm{Rb})$ and drives cell-cycle progression, a process inhibited by p16 (fig. 2). When $\mathrm{Rb}$ is phosphorylated, the inhibition of the transcription factor E2F, which leads to progression of cells through the cell cycle, is lost. This pathway is frequently disrupted in breast cancer through p16 loss, CDK4/6 amplification, cyclin D overexpression, or Rb loss, leading to inactivation of the G1-S phase checkpoint.

In cancer cells, since the pathway described above is known to be frequently activated, efforts have been made to block it pharmacologically [27]. The first-generation CDK inhibitors such as flavopiridol were nonselective CDK inhibitors that blocked CDK4 but also had significant side effects. However, in recent years several selective CDK4/6 inhibitors have been developed and have entered clinical studies. The most advanced drug in this class is PD0332991 or palbociclib (Pfizer Inc.). Palbociclib is a potent oral inhibitor of CDK4 and CDK6 that prevents downstream phosphorylation of $\mathrm{Rb}$, thereby leading to $\mathrm{G} 1$ arrest. In an attempt to identify predictors of response, Finn and co-workers examined the in vitro sensitivity to PD-0332991 across a panel of 47 molecularly characterized human breast cancer cell lines [28]. They determined the growth inhibition for each cell line and compared it with baseline gene expression data to identify genes associated with PD0332991 response. Cell lines representing luminal ER-positive subtype were the most sensitive to growth inhibition by PD-0332991, while non-luminal/basal subtypes were the most resistant. Alto- 
gether 450 genes were differentially expressed between sensitive and resistant tumours. As expected, Rb and cyclin D1 were elevated and p16 was decreased in the most sensitive lines. These in vitro results represented a strong rationale for the treatment of ERpositive breast cancer patients.

To proceed with the clinical development of this drug, a phase I trial investigated dose-limiting toxicity (DLT) and maximum tolerated dose (MTD) of the first-in-class oral CDK4/6 inhibitor PD0332991 administered once daily for 21 of 28 days in patients with $\mathrm{Rb}$-positive advanced solid tumours. This phase I study led to the conclusion that PD-0332991 warranted phase II testing at $125 \mathrm{mg}$ once daily, a dose at which neutropenia was the sole significant toxicity [29]. As a next step, 37 patients with advanced breast cancer positive for $\mathrm{Rb}$ were enrolled in a single-arm phase II trial. The majority of the patients (84\%) were HR positive and HER2 negative. Patients had received a median of 2 prior cytotoxic therapies. Palbociclib showed clinical activity in this heavily pretreated cohort of breast cancer patients with a clinical benefit rate of $19 \%$ overall. Median PFS overall was 3.7 months, but significantly longer for those with HR-positive versus HR-negative disease $(\mathrm{p}=0.03)$ and those who had previously progressed through endocrine therapy for advanced disease $(\mathrm{p}=0.02)$. The major toxicity was neutropenia ( $51 \%$ grade III/IV), which could be easily managed with dose reduction. However, neither analysis of p16 nor cyclin D identified a sensitive population [30].

Building on the encouraging results of palbociclib in HR-positive breast cancer, an open-label randomized phase II trial enrolled 165 postmenopausal ER-positive and HER2-negative breast cancer patients who had not received any systemic therapy for advanced disease [31]. Patients were randomized either to letrozole alone or to letrozole + palbociclib (125 mg, given once daily for 3 weeks followed by 1 week off over 28-day cycles). Patients were enrolled sequentially in 2 separate cohorts: in cohort 1, patients were enrolled on the basis of their ER-positive and HER2-negative biomarker status alone, whereas in cohort 2 they were also required to have amplification of cyclin D1 or loss of $\mathrm{p} 16^{\mathrm{INK} 4 \mathrm{~A}}$. Median PFS was 10.2 months for the letrozole group and 20.2 months for the palbociclib plus letrozole group (HR 0.488, 95\% CI 0.319-0.748; $\mathrm{p}=0.0004$ ). Grade III/IV neutropenia was reported in $54 \%$ in the palbociclib plus letrozole group versus $1 \%$ in the letrozole group. However, no cases of febrile neutropenia were reported. Building on this substantial improvement of PFS with the addition of palbociclib to letrozole, the FDA granted accelerated approval to palbo- ciclib (IBRANCE, Pfizer Inc.) for use in combination with letrozole for the treatment of postmenopausal women with ER-positive HER2-negative advanced breast cancer as initial endocrine-based therapy for their metastatic disease.

\section{New Therapeutic Options, New Challenges}

To ensure adequate safety in the use of these innovative and promising therapeutic options, it is important to pay attention to the new range of side effects of these small molecules. Possible specific side effects of mTOR inhibitors are stomatitis, diarrhoea, dyspnoea, non-infectious/interstitial pneumonitis, hyperglycaemia, fatigue and rash [32]. The number of adverse-event-related on-treatment deaths is particularly evident in patients above 65 years [20], therefore the FDA recommends strict indications especially for elderly patients. Moreover, for PI3K inhibitors mood changes have been described as a further serious side effect, which can be connected to a possible crossing of the blood-brain barrier [33-35]. The major toxicity of palbociclib is neutropenia [31]. A further challenge is selecting the ideal patient population. More recently, the questions of which biomarkers would be useful predictive factors and in which clinical situation dual inhibition would be most effective have been controversially discussed $[36,37]$.

\section{Conclusion}

The results clearly show that inhibition of the PI3K/AKT/ mTOR pathway and CDK4/6 are promising ways to improve the efficacy of endocrine treatment in ER-positive breast cancer patients with comparably few side effects. Further studies have to deal with advanced strategies to overcome endocrine resistance. Due to the heterogeneity of breast cancer, translational approaches are necessary in order to define suitable patient cohorts and predictive biomarkers for a personalised therapy with a high therapeutic index.

\section{Disclosure Statement}

K. Almstedt has nothing to disclose. M. Schmidt received honoraria from AstraZeneca, Novartis, and Pfizer.

\section{References}

1 Perou C, Sorlie T, Eisen M, et al.: Molecular portraits of human breast tumours. Nature 2000;406:747-752.

2 Lindström LS, Karlsson E, Wilking UM, et al.: Clinically used breast cancer markers such as estrogen receptor, progesterone receptor, and human epidermal growth factor receptor 2 are unstable throughou tumor progression. J Clin Oncol 2012;30:2601-2608.

Targeted Therapies Overcoming Endocrine

Resistance in HR-Positive BC
3 Partridge AH, Rumble RB, Carey LA, et al.: Chemotherapy and targeted therapy for women with human epidermal growth factor receptor 2-negative (or unknown) advanced breast cancer. American Society of Clinical Oncology Clinical Practice Guideline. J Clin Oncol 2014;32:3307-3329.

4 Cardoso F, Costa A, Norton L, et al.: ESO-ESMO 2nd international consensus guidelines for advanced breast cancer (ABC2). Ann Oncol 2014;25:1871-1888.
5 Harbeck N, Scharl A, Thomssen C, Muller V: AGO recommendations for diagnosis and treatment of patients with advanced and metastatic breast cancer: Update 2013. Breast care 2013;8:181-185.

6 Osborne CK, Shou J, Massarweh S, Schiff R: Crosstalk between estrogen receptor and growth factor receptor pathways as a cause for endocrine therapy resistance in breast cancer. Clin Cancer Res 2005; 11:865-870. 
7 Fagan DH, Yee D: Crosstalk between IGF1R and estrogen receptor signaling in breast cancer. J Mammary Gland Biol Neoplasia 2008;13:423-429.

8 Cerliani JP, Guillardoy T, Giulianelli S, et al.: Interaction between FGFR-2, STAT5, and progesterone receptors in breast cancer. Cancer Res 2011;71:37203731.

9 Roskoski R Jr: The ErbB/HER family of protein-tyrosine kinases and cancer. Pharmacol Res 2014;79:34-74.

10 Johnston S, Pippen J Jr, Pivot X, et al.: Lapatinib combined with letrozole versus letrozole and placebo as first-line therapy for postmenopausal hormone receptor-positive metastatic breast cancer. J Clin Oncol 2009;27:5538-5546.

11 Kaufman B, Mackey JR, Clemens MR, et al.: Trastuzumab plus anastrozole versus anastrozole alone for the treatment of postmenopausal women with human epidermal growth factor receptor 2-positive, hormone receptor-positive metastatic breast cancer: Results from the randomized phase III TAnDEM study. J Clin Oncol 2009;27:5529-5537.

12 Burstein HJ, Cirrincione CT, Barry WT, et al.: Endocrine therapy with or without inhibition of epidermal growth factor receptor and human epidermal growth factor receptor 2: A randomized, double-blind, placebo-controlled phase III trial of fulvestrant with or without lapatinib for postmenopausal women with hormone receptor-positive advanced breast cancerCALGB 40302 (Alliance). J Clin Oncol 2014;32:39593966.

13 Cancer Genome Atlas Network: Comprehensive molecular portraits of human breast tumours. Nature 2012;490:61-70.

14 Miller TW, Hennessy BT, González-Angulo AM, et al.: Hyperactivation of phosphatidylinositol-3 kinase promotes escape from hormone dependence in estrogen receptor-positive human breast cancer. J Clin Invest 2010;120:2406-2413.

15 Wander SA, Hennessy BT, Slingerland JM: Next-generation mTOR inhibitors in clinical oncology: How pathway complexity informs therapeutic strategy. J Clin Invest 2011;121:1231-1241.

16 United States Food and Drug Administration: FDA approves Afinitor for advanced breast cancer. http:// www.fda.gov/NewsEvents/Newsroom/PressAnnouncements/ucm312965.htm retrieved 02.03.15.
17 Baselga J, Campone M, Piccart M, et al.: Everolimus in postmenopausal hormone-receptor-positive advanced breast cancer. N Engl J Med 2013;366:520-529.

18 Yardley DA, Noguchi S, Pritchard KI, et al.: Everolimus plus exemestane in postmenopausal patients with $\mathrm{HR}(+)$ breast cancer: BOLERO-2 final progressionfree survival analysis. Adv Ther 2013;30:870-884.

1919 Beck JT, Hortobagyi GN, Campone M, et al.: Everolimus plus exemestane as first-line therapy in HR(+), HER2() advanced breast cancer in BOLERO-2. Breast Cancer Res Treat 2014;143:459-467.

20 Piccart M, Hortobagyi GN, Campone M, et al.: Everolimus plus exemestane for hormone-receptor-positive, human epidermal growth factor receptor-2-negative advanced breast cancer: overall survival results from BOLERO-2†. Ann Oncol 2014;25:2357-2362.

21 Bachelot T, Bourgier C, Cropet C, et al.: Randomized phase II trial of everolimus in combination with tamoxifen in patients with hormone receptor-positive, human epidermal growth factor receptor 2-negative metastatic breast cancer with prior exposure to aromatase inhibitors: a GINECO study. J Clin Oncol 2012; 30:2718-2724.

22 Wolff A, Lazar A, Bondarenko I, et al.: Randomized phase III placebo-controlled trial of letrozole plus oral temsirolimus as first-line endocrine therapy in postmenopausal women with locally advanced or metastatic breast cancer. J Clin Oncol 2013;31:195-202.

23 ClinicalTrials.gov identifier: NCT01610284.

24 ClinicalTrials.gov identifier: NCT01633060.

25 Hanahan D, Weinberg RA: The hallmarks of cancer. Cell 2000;100:57-70.

26 Malumbres M, Barbacid M: To cycle or not to cycle: A critical decision in cancer. Nat Rev Cancer 2001;1: 222-231.

27 Dickson MA: Molecular pathways: CDK4 inhibitors for cancer therapy. Clin Cancer Res 2014;20:3379-3383.

28 Finn RS, Dering J, Conklin D, et al.: PD 0332991, a selective cyclin D kinase 4/6 inhibitor, preferentially inhibits proliferation of luminal estrogen receptor-positive human breast cancer cell lines in vitro. Breast Cancer Res 2009;11:R77.
29 Flaherty KT, Lorusso PM, DeMichele A, et al.: Phase I, dose-escalation trial of the oral cyclin-dependent kinase 4/6 inhibitor PD 0332991, administered using a 21-day schedule in patients with advanced cancer. Clin Cancer Res 2012;18:568-576.

30 DeMichele A, Clark AS, Tan KS, et al.: CDK 4/6 inhibitor palbociclib (PD0332991) in Rb+ advanced breast cancer: Phase II activity, safety, and predictive biomarker assessment. Clin Cancer Res 2015;21:995-1001.

31 Finn RS, Crown JP, Lang I, et al.: The cyclin-dependent kinase $4 / 6$ inhibitor palbociclib in combination with letrozole versus letrozole alone as first-line treatment of oestrogen receptor-positive, HER2-negative, advanced breast cancer (PALOMA-1/TRIO-18): A randomised phase 2 study. Lancet Oncol 2015;16:25-35.

32 Chia S, Gandhi S, Joy AA, et al.: Novel agents and associated toxicities of inhibitors of the PI3K/Akt/mTOR pathway for the treatment of breast cancer. Curr Oncol 2015;22:33-48.

33 Nanni P, Nicoletti G, Palladini A, et al.: Multiorgan metastasis of human HER-2+ breast cancer in Rag2-/;Il2rg-/- mice and treatment with PI3K inhibitor. PLoS One 2012; 7:e39626.

34 Maira, S., Pecchi, S., Huang A, et al.: Identification and characterization of NVP-BKM120, an orally available pan-class I PI3-kinase inhibitor. Mol Cancer Ther 2012;11:317-328.

35 Peddi P, Hurvitz S: PI3K pathway inhibitors for the treatment of brain metastases with a focus on HER2+ breast cancer. J Neurooncol 2014;117:7-13.

36 Baselga J, Semiglazov V, van Dam P, et al.: Phase II randomized study of neoadjuvant everolimus plus letrozole compared with placebo plus letrozole in patients with estrogen receptor-positive breast cancer. J Clin Oncol 2009;27:2630-2637.

37 Ellis MJ, Lin L, Crowder R, et al.: Phosphatidyl-inositol-3-kinase alpha catalytic subunit mutation and response to neoadjuvant endocrine therapy for estrogen receptor positive breast cancer. Breast Cancer Res Treat 2010;119:379-390. 